\title{
Driver anger on the information superhighway: A content analysis of online complaints of offensive driver behavior
}

\author{
Wickens, C. M., Wiesenthal, D. L., Hall, A., \& Roseborough, J.
}

Version Post-Print/Accepted Manuscript

Citation Wickens, C. M., Wiesenthal, D. L., Hall, A., \& Roseborough, J. (2013).

(published version) Driver anger on the information superhighway: A content analysis of online complaints of offensive driver behaviour. Accident Analysis and Prevention, 51, 84-92.

\section{Copyright / License (c) (i)}

This work is licensed under a Creative Commons AttributionNonCommercial-NoDerivatives 4.0 International License.

Publisher's Statement The final publication is available at Elsevier via http://dx.doi.org/10.1016/j.aap.2012.10.007.

Always cite the published version, so the author(s) will receive recognition through services that track citation counts, e.g. Scopus. If you need to cite the page number of the TSpace version (original manuscript or accepted manuscript) because you cannot access the published version, then cite the TSpace version in addition to the published version using the permanent URI (handle) found on the record page. 
Running head: DRIVER ANGER ON THE INFORMATION SUPERHIGHWAY

Driver Anger on the Information Superhighway:

A Content Analysis of Online Complaints of Offensive Driver Behaviour

Christine M. Wickens ${ }^{1}$, David L. Wiesenthal ${ }^{2}$, Ashley Hall ${ }^{2}, \&$ James Roseborough $^{2}$

${ }^{1}$ Centre for Addiction and Mental Health, Toronto, Ontario, Canada

${ }^{2}$ York University, Toronto, Ontario, Canada

Corresponding Author:

Christine M. Wickens

Centre for Addiction and Mental Health

Social and Epidemiological Research

33 Russell Street, T417

Toronto, ON M5S 2S1

Phone: (416) 535-8501 Ext. 4711

Fax: (416) 595-6899

E-mail: Christine.Wickens@ camh.ca 


\begin{abstract}
In recent years, several websites have been developed allowing drivers to post their complaints about other motorists online. These websites allow drivers to describe the nature of the offensive behaviour and to identify the offending motorist by vehicle type, colour, and license plate number. Some websites also ask drivers to list the location where the event took place and the exact date and time of the offence. The current study was a content analysis of complaints posted to RoadRagers.com between 1999 and 2007 ( $N=5624)$. The purpose of the study was to: (1) assess the research value of this novel data source; (2) demonstrate the value of content analysis to the study of driver behaviour; (3) further validate an existing coding scheme; (4) determine whether this new data source would replicate previous research findings regarding the most frequent types of driver complaints and temporal distribution of these reports; (5) provide recommendations for improved driver training and public safety initiatives based on these data. A coding scheme that was originally developed for an assessment of complaints submitted to the Ontario Provincial Police (OPP) (Wickens et al., 2005) was revised to accommodate the new dataset. The inter-rater reliability of the revised coding scheme as applied to the website complaints was very good (kappa $=.85)$. The most frequently reported improper driver behaviours were cutting/weaving, speeding, perceived displays of hostility, and tailgating. Reports were most frequent on weekdays and during the morning and afternoon rush hour. The current study replicated several findings from the analysis of reports to the OPP, but possible differences in the sample and data collection method also produced some differences in findings. The value of content analysis to driver behaviour research and of driver complaint websites as a data source was demonstrated. Implications for driver safety initiatives and future research will be discussed.
\end{abstract}

Keywords: Driver anger, driver aggression, content analysis 


\section{Introduction}

\subsection{Driver anger: precursor to driver aggression}

Driver aggression is a major risk to highway safety, and has been estimated to be involved in $50 \%$ of all motor vehicle collisions (Snyder, 1997). Definitions of aggressive driving are extremely varied (Tasca, 2000), but for the purposes of the current discussion, we will define aggressive driving as roadway behaviour that shows either deliberate or unintentional disregard for the safety, well-being, and/or convenience of other drivers. Numerous factors internal to the driver (e.g., personality [Lajunen and Parker, 2001; Matthews et al., 1991], mood [Groeger, 1997; Novaco, 1991], and the experience of stress [Hennessy and Wiesenthal, 1999, 2001a]) have been identified as contributors to driver aggression. Yet, perhaps the most proximal cause of, or precursor to, driver aggression is anger. Much of the aggressive driving behaviour seen on the roadways is the result of driver anger and retaliation for a perceived driving offence (Gulian et al., 1989; Neighbors et al., 2002; Parker et al., 2002). Although not all driver anger results in aggression, the relationship is certainly a strong one (e.g., Deffenbacher et al., 2003; Wickens et al., 2011). A recent meta-analysis of 28 independent studies reported an average correlation of .40 between anger and aggressive driving (Nesbit et al., 2007).

Attempts to approximate the frequency of roadway anger have produced a wide range of estimates, but all suggest that it is a relatively common roadway occurrence. In fact, anger when driving is more common than anger when engaged in other everyday activities (Parkinson, 2001). In a diary study where drivers were equipped with microcassette recorders, approximately $85 \%$ of participants reported experiencing anger at least once during a 2-week period, and overall, drivers expressed anger in about one fifth of all trips taken (Underwood et al., 1999). In a similar study conducted over a 10-day period, drivers reported incidents of driver anger on slightly more than a daily basis (Neighbors et al., 2002). The anger only lasted a few minutes but was reported 
as being relatively intense. In another study where participants drove an instrumented vehicle and reported their emotions aloud to the passenger experimenter over a 50-min period, anger was reported an average of 1.5 times (Mesken et al., 2007).

\subsection{Methodologies used to identify objectionable roadway behaviours}

Given the frequency of driver anger, and its strong impact on both driver aggression and resulting motor vehicle collisions, several research initiatives have been undertaken to identify the types of objectionable roadway behaviours that cause driver anger. These research initiatives have adopted a wide variety of predominantly quantitative methodologies. For instance, questionnaire studies (e.g., Deffenbacher et al., 1994; Hennessy and Wiesenthal, 2001b, Wiesenthal et al., 2000) have presented drivers with a list of possible driving altercations and asked them to rate their anticipated anger and/or aggression in response to these events. Largescale telephone surveys conducted by national organizations (e.g., Traffic Injury Research Foundation; Vanlaar et al., 2008) have adopted a similar strategy but have utilized larger and more nationally representative samples. Field experimentation has been conducted using multiple techniques. A prime example is the previously mentioned study where participants drove an instrumented vehicle and were accompanied by a passenger experimenter to whom they verbally reported their emotions (Mesken et al., 2007). In other field studies, drivers' level of stress has been assessed in situ over a cellular telephone (e.g., Hennessy and Wiesenthal, 1999, 2001b; Wickens and Wiesenthal, 2005). Driving diary studies, like the ones previously described (Neighbors et al., 2002; Underwood et al., 1999), have also been used to identify angerprovoking driving events.

Occasionally, these studies have identified a specific behaviour as anger-provoking. For instance, the large-scale survey conducted by TIRF reported that participants were most concerned about red-light running, street racing, and excessive speeding (Vanlaar et al., 2008). 
Likewise, Parkinson (2001) found that respondents most frequently reported their most recent episode of driving anger as involving either: (1) another driver cutting in or pulling out in front of them, or (2) other drivers retaliating (e.g., tailgating and hostile gestures) for perceived bad driving on the part of the participant. On the other hand, many of these studies have categorized specific behaviours into classes of anger-provoking events based on established theory or the results of factor analysis. For instance, in their assessment of appraisal theory, Mesken et al. (2007) reported that anger while driving was most often elicited by events involving impeded progress and, sometimes, safety-related events involving another driver. In the development of their driving anger scale, Deffenbacher et al. (1994) factor analyzed anger ratings of several types of driving events. They concluded that women were most angered by events that interfered with their driving (e.g., speeding, running a stop sign, and road repairs), whereas men were most angered by authority on the roadways (e.g., presence of police) and slow driving. Neighbors et al. (2002) summarized anger-provoking events from their diary study as falling into the categories of discourtesy and dangerous driving, whereas Underwood et al. (1999) reported that anger experienced by their diary study participants usually occurred following a near accident.

Each of the methodologies incorporated by these studies has their strengths and weaknesses. For instance, questionnaire studies facilitate comparison of various types of angerprovoking driving encounters, but may fail to include some potentially upsetting driving events. Large-scale telephone surveys allow for a highly representative population-level analysis, but are limited in the amount of detailed response that can be solicited in short but expensive interviews. In-vehicle studies maximize external validity, but the presence of an in-vehicle experimenter may alter the nature of the driving experience. Nonetheless, when the findings of these various methodologies are combined and triangulated, they provide a comprehensive picture of the various sources of driver anger. These methodologies may also highlight possible differences in 
the type of driving events considered anger-provoking due to the gender of the victim driver (Deffenbacher et al., 1994; Parker et al., 2002), the culture in which the altercation took place (Parker et al., 2002), and circumstances of the event that might mitigate or exacerbate the resulting anger (Wickens et al., 2011).

Research initiatives using more qualitative methodologies to identify objectionable roadway behaviours that elicit driver anger are more atypical. Organizations such as the National Highway Traffic Safety Administration have conducted focus groups with drivers from a specific geographical location in order to identify driving behaviours considered offensive by local motorists (National Highway Traffic Safety Administration, 1998). Perhaps an even less commonly used qualitative methodology is content analysis. This data analysis technique derives inferences by "systematically and objectively identifying specific characteristics of messages" (Whitley, 1996, p.385). This methodology has generally been applied to speeches, letters, pictorial content, and news reports; however, it has also been used to analyze police databases (Sarkar et al., 2000; Wickens et al., 2005).

Wickens et al. (2005) developed a ten-category coding scheme and content-analyzed complaints of improper driver behaviour received by the Ontario Provincial Police (OPP) in the year 2000. The analysis revealed that the most frequently reported complaints involved dangerous lane changes/improper lane usage, speeding, and tailgating. It may be that these were the most frequently occurring offensive driver behaviours, or that they were perceived as the most offensive and thus warranted reporting to the authorities. The latter interpretation suggests that drivers experience a sense of discomfort when their ability to predict the behaviour of other motorists is threatened by violations of crucial driving norms. The study also found that complaints were more frequently reported on Fridays and less frequently reported on Mondays and Sundays. Complaints were most frequently reported during the afternoon rush hour (1500 - 
1800 hours) and were most prevalent during the summer months when motorists are traveling both for work and vacations. Together, these results clearly demonstrated that increased traffic congestion is related to the number of reported incidents of offensive driver behaviour.

As with other research methodologies, content analysis has both its strengths and its weaknesses. Content analysis allows for the systematic investigation of existing archival data, which are high in external validity. However, the results of content analysis can be strongly influenced by the weaknesses of the data source. For instance, complaints submitted to the OPP are screened by personnel in the OPP Call Centre, who record the most relevant information before radio calling to officers on the road. This screening of reports may result in the systematic elimination of pertinent data. For this reason, content analysis coding schemes should be applied to a variety of data sources in order to strengthen the reliability and interpretability of their results.

\subsection{The current study}

The current study is an extension of the content analysis conducted by Wickens et al. (2005). In recent years, several websites have been developed allowing drivers to post their complaints about motorists online. These websites allow drivers to describe the nature of the offensive behaviour and to identify the offending motorist by vehicle type, colour, and license plate number. Some websites also ask drivers to list the location where the event took place and the exact date and time of the offence. To the best of our knowledge, this new form of communication has not yet been content-analyzed, and would serve as an excellent data source for further assessment of the reliability of Wickens et al.'s findings (2005).

The current study focused on complaints posted to RoadRagers.com. Wickens et al.'s (2005) content analysis coding scheme that was originally developed for an assessment of complaints submitted to the OPP was revised slightly to accommodate the new dataset. The 
purpose of the current study was: (1) to assess the research value of this novel data source (i.e., complaints to an online website); (2) to demonstrate the value of content analysis, an under-used qualitative research methodology, to the study of driver behaviour; (3) to further validate an existing coding scheme; (4) to determine whether this new data source would replicate previous research findings regarding the most frequent types of driver complaints, as well as the month of year, day of week, and time of day when these complaints are most frequently reported; (5) to provide recommendations for improved driver training and public safety initiatives based on these data.

\section{Methods}

\subsection{Sample}

According to its disclaimer, the RoadRagers.com website was created as "a safe environment for frustrated drivers to vent disapproval of what they witness on the streets and highways". Visitors to the site are asked to provide details of events involving alleged "bad drivers", including what transpired, where and when it took place, and the make, model, colour, and licence plate number of the offending vehicle. The site also allows visitors to see a listing of previous complaints by year of submission. The current study focuses on the complaints posted to RoadRagers.com that were available for download in May 2007. There were 5624 complaints dating back to July 1999.

\subsection{Coding scheme}

The coding scheme developed by Wickens et al. (2005) was adopted for the current study. This coding scheme was originally used to assess complaints submitted to the OPP; however, as the OPP are primarily responsible for provincial highways, the existing coding scheme did not include behaviours that would occur on major arterial or minor roads (e.g., running red lights or stop signs). Therefore, the coding scheme needed to be revised slightly for the current dataset. 
The revised coding scheme included eleven main categories and several sub-categories. A listing of these categories and abbreviated definitions are provided in Table 1. The full coding scheme, including instructions and examples provided for the coders, can be obtained from the authors.

Several other revisions to the coding scheme were made to improve the quality of the results. Specifically, several sub-categories were introduced in order to provide a more detailed understanding of driver complaints. Dangerous lane changes and lane usage was the most frequently reported complaint to the OPP (Wickens et al., 2005). For the current study, two subcategories were created so that improper lane usage (e.g., not staying within the lane markings and passing in a no-passing zone) could be differentiated from weaving and cutting off. Likewise, although it was not formally measured and reported in Wickens et al.'s study, the improperly equipped/unsafe vehicle category often reflected a lack of turn signal use. The current study included sub-categories so that coders could note whether the failing equipment was a missing or unused turn signal versus something else. In the Wickens et al. study, the hostile driver display category included a wide range of behaviours including more minor actions such as yelling and obscene gestures as well as more extreme actions such as physically assaulting another driver or pointing a gun. The current study introduced two sub-categories of hostile versus violent driver displays. Finally, the driver inattention category was sub-divided into cell-phone use versus other types of distractions.

The final revision to the coding scheme was the addition of categories to reflect blocking behaviour (i.e., preventing other vehicles from merging or passing) and erratic/improper braking, which were common in the current dataset. Complaints to the OPP Call Centre are received and recorded by personnel who then communicate the information to officers in the field. It is possible that in this process, the complaints are filtered and screened in such a way that behaviours not specifically in violation of the Highway Traffic Act, such as blocking or erratic 
braking, are not consistently recorded. Since the current dataset consists of reports submitted and recorded by the victim drivers themselves, it was necessary to add to the coding scheme categories reflecting offensive, but not illegal behaviour.

\subsection{Procedure}

The modified coding scheme was initially applied to a sub-sample of the RoadRagers.com dataset (July 1999 to December 2000; $n=312$ ), and was repeatedly revised until an adequate measure of inter-rater reliability between two coders was attained (kappa $=.75$; Altman, 2005 as cited in Shin et al., 2005). The coding scheme was then applied to the remaining sample. All reports were coded by two primary coders. Each report could be placed in one or

more categories (or sub-categories). For example, the complaint "tailgating, trying to pass on the shoulder, cut off cars, honking horn, flipping the bird, excessive speed" was classified as tailgating (B), improper lane usage (Ci), weaving/cutting (Cii), perceived hostile driver display (Hi), and speeding/racing (Ai). If there was a disagreement between the two primary coders, a third independent coder categorized the complaint. The final classification included the categories selected by at least two of the three coders. Upon completion of all coding, the three coders met to discuss and come to an agreement on classification of the cases where at least one category was not selected by two of the three coders.

\section{Results}

The RoadRagers.com archive included 5624 reports. Of these, 120 reports originated from outside North America and were deleted from the dataset. Five complaints were deleted because they did not describe another driver's behaviour (e.g., reports of one's own poor driving behaviour, denials of others' accusations, etc.). There were 181 reports that were obvious duplicates of reports already included in the archive, and thus were deleted. In three cases, there were two reports that identified different aspects of the same event. In these cases, the two reports 
were combined into one. Therefore, the results of the analysis are based on the remaining 5315 reports.

Although no demographic information concerning the complainants was available from the website, participants were asked to indicate in what state or province the offensive driving events took place. A total of 4988 reports (93.8\%) were identified as taking place in the United States, and 298 reports $(5.6 \%)$ were identified as taking place in Canada. The top states in which driving events were reported were Texas (750 reports; $14.1 \%$ ), California (716 reports; 13.5\%), Illinois (484 reports; 9.1\%), Arizona (288 reports, 5.4\%), and Florida (223 reports; 4.2\%). Ontario was the province with the greatest number of driving reports in Canada (135 reports; 2.5\%), followed by British Columbia (50 reports; $.9 \%$ ).

The reliability of the coding scheme across these reports was very good (kappa $=.85$; Altman, 2005 as cited in Shin et al., 2005). The three coders could not reach an agreement on the classification of just 28 complaints, less than $.01 \%$ of the total sample. These reports were discussed and coded by the three coders as a group.

Several corrections to the reports were necessary. In some instances, participants had identified the event as occurring at a particular time, day, month, or year from a drop-down list on the website, but had provided an alternative time, day, month, or year in their written description of the event. Contradictions in the time $(n=5)$, day $(n=2)$, and month $(n=1)$ of the incident were rare, and were interpreted as entry errors. The information given in the written description was taken as valid.

Contradictions in the year of the reported event were more problematic. In the early years of the archive (i.e., 2000-2004), there were a few January reports appearing at the end of each year's data file, likely because the website had not yet provided the new year as an answer option for drivers submitting a complaint. However, in later years of the archive, this problem was more 
severe. In the 2005 file, 199 reports from a mixture of months could be found at the end of the data file, and in many of these reports the respondents clearly indicated that the actual year of the event (usually 2006 or 2007) was not listed as an answer option. In addition, in the 2006 data file, several weeks would go by without a report being submitted, when trends would have suggested that a dozen or more reports should have been expected each day during that time period. These patterns in the dataset suggested that the website had experienced technical difficulties throughout the latter years of its operation. A total of 251 reports had the year of the event corrected. Most of these reports were taken from 2005 and were re-labeled 2006 or 2007. These problems with the dataset were not expected to influence the analysis of the time of day or the day of week when drivers experienced driver anger. However, it was recognized a priori that these problems with the dataset may jeopardize the analysis of monthly reporting trends.

\subsection{Classification of complaints}

As can be seen in Fig. 1, the most frequently reported driver behaviour was weaving and cutting, which was included in 54\% of all complaints. This was followed by speeding (29\% of all complaints), perceived hostile driver displays ( $25 \%$ of all complaints), and tailgating (24\% of all complaints).

\subsection{Complaints as a function of month of year}

The remaining analyses examine reporting trends as a function of month of year, day of week, and time of day. For the analysis of monthly reporting trends only, a reduced dataset was used. This was done for several reasons. First, the 1999 archive began in July of that year, making an analysis of reporting trends across the year impossible. Second, the number of reports in each remaining year of the archive increased significantly, suggesting that any analysis of reporting trends across the year may be influenced by the website's increasing popularity over time. The data from 1999 and 2000 were the most vulnerable to the potential influence of the 
website's increasing popularity throughout the year. Third, it was anticipated that the data problems found in the latter years of the archive may interfere with or bias the examination of monthly reporting trends. For these reasons, the analysis of monthly reporting trends was conducted only on the 2001 through 2004 archives $(n=2447)$.

As can be seen in Fig. 2, the number of complaints varied by month $\left(\chi^{2}(11)=32.73, p<\right.$ .001), however, there was no definitive seasonal pattern in the results. The four months with the highest frequency of reports were July through October. Four of the 5 months with the lowest frequency of reports were November through February.

\subsection{Complaints as a function of day of week}

As can be seen in Fig. 3, the frequency of complaints varied by day of the week $\left(\chi^{2}(6)=\right.$ 431.18, $p<.001)$. The number of complaints was greatest on weekdays, although Tuesday (18.5\% of all complaints), Wednesday (17.5\% of all complaints), and Thursday (17\% of all complaints) generated the greatest number of complaints overall. The number of complaints filed on weekends was much lower, with Sundays generating the lowest number of complaints overall ( $8 \%$ of all complaints). The weekday/weekend distinction was generally stable for each category of complaints, with the exception of perceived violent driver displays. In that case, Mondays were included among the days with the lowest number of complaints (12\% of the violent display reports), along with Saturdays (10\% of these reports) and Sundays (13\% of these reports).

\subsection{Complaints as a function of time of day}

As can be seen in Fig. 4, the number of complaints varied by time of day $\left(\chi^{2}(7)=\right.$ $3126.74, p<.001)$. The distribution is clearly bimodal, with the greatest number of complaints originating from the $0600-0859 \mathrm{~h}$ timeslot and the $1500-1759 \mathrm{~h}$ timeslot. This trend holds for all categories of complaints.

\section{Discussion}




\subsection{Classification of complaints}

The classification of behaviour type for the RoadRagers.com complaints were generally consistent with content analyses of complaints submitted to police departments (Sarkar et al., 2000) and driving diaries (Wickens et al., 2012). In the current study, weaving and cutting was the most commonly reported behaviour ( $54 \%$ of all complaints), followed by speeding ( $29 \%$ of all complaints), perceived hostile driver displays (25\% of all complaints), and tailgating (24\% of all complaints). These results are consistent with the content analysis of complaints submitted to the OPP, which used the same basic coding scheme. Results of that study identified dangerous lane usage (involved in $62 \%$ of all complaints received by the OPP), speeding ( $35 \%$ of all complaints), and tailgating (18\% of all complaints) as the driver behaviours most frequently reported to the OPP (Wickens et al., 2005). This general replication of results provides further validation for the structure and utility of the coding scheme. Interestingly, in the study of OPP complaints, dangerous lane usage included weaving and cutting as well as improper lane usage (e.g., not staying within the lane markings and passing in a no-passing zone) in a single category. In the current study, these two driver behaviours were differentiated. Nonetheless, weaving and cutting remained the top driver complaint.

The most significant difference in the classification of complaints between the two data sources is the percentage of complaints involving perceived displays of driver hostility. In the OPP study (Wickens et al., 2005), only 6\% of complaints involved perceived hostile/violent driver displays. In the current study, driver hostility and driver violence were divided into two separate categories, and still, perceived driver hostility was involved in $25 \%$ of reports. It is likely that this increase in the number of reported cases of driver hostility is a function of how each of the datasets was collected. In the case of the OPP data, it is likely that perceived driver hostility was screened out of the reports by the OPP Call Centre personnel. As obscene gestures, unkind 
language, and rudeness are not illegal, this information may have been considered irrelevant for the officers in the field and thus deleted from the official record of the call. This type of screening would have resulted in an underestimation of the frequency of hostile driver displays. In the case of the RoadRagers.com data, the name of the website suggests that it is specifically soliciting reports of hostile or aggressive roadway behaviour. Thus, it is likely that this dataset overestimates the frequency of hostile and violent driver displays.

It should also be noted that none of the complaints was coded as a hazardous road condition that was not attributable to driver behaviour $(\mathrm{J})$ and that this category has not been included in any of the figures. Although reports of roadway hazards may be made to the OPP, such reports are unlikely to appear on a driver complaint website.

\subsection{Monthly reporting trends}

Although there was no definitive seasonal pattern among the RoadRagers.com complaints, the four months with the highest frequency of reports were July through October and 4 of the 5 months with the lowest frequency of reports were November through February. It is tempting, therefore, to conclude that the results of the current study replicated the summer peak and winter decline found by previous studies. For instance, Rathbone and Huckabee (1999) surveyed American organizations that have implemented programs to combat aggressive driving (e.g., police departments, state department of transportation, and metropolitan planning organizations) and, although their sample was quite small (i.e., 40 respondents, 80 incidents of aggressive driving), they found a peak in the summer months and a decline in the winter months. Wickens et al. (2005) reported that complaints to the OPP followed a similar pattern. In the current study, however, the differences in frequency across the year were very small, and the lowest number of reports was found in June, typically considered a summer month. Furthermore, the seasonal trend was not stable across each category of complaints. For these reasons, we 
hesitate to conclude that the seasonal trend was replicated.

There are several reasons why the monthly reporting trends found in the current study are not as definitive as those found in the study of complaints to the OPP (Wickens et al., 2005). First, it is possible that the strength of the effect is not strong enough to be found with this reduced dataset. The OPP dataset included 14406 complaints. Still, the Rathbone and Huckabee report (1999) also found a peak in summer reporting and a decline in winter reporting with only 80 incidents of aggressive driving. Second, the OPP dataset was collected in a climate where the winter season is associated with inclement weather. The RoadRagers.com dataset included complaints from all over the United States and Canada, including regions where winter is not associated with ice and snow. It is possible that the seasonal reporting trends are only found in colder climates. The fact that Texas, California, Arizona, and Florida were among the five states with the greatest number of online complaints submitted (accounting for nearly $37 \%$ of the reported events included in the monthly trends analysis) supports this suggestion. Third, the difference may be a result of the data collection technique. In the OPP study, complaints were submitted immediately by drivers using their cellular telephones to contact the police. With this more convenient means of reporting, it is likely that motorists reported events that occurred when driving for work as well as when driving for pleasure. In the current study, complaints could only be submitted later, when drivers had access to the Internet. As will be described subsequently, the current sample of drivers likely consisted of daytime workers who had access to the Internet at their place of employment and were reporting anger-provoking events that they encountered while driving for work only. This interpretation of the sample's characteristics is supported by the reporting trends for day of the week and time of the day.

\subsection{Daily reporting trends}

The number of complaints to the RoadRagers.com website was greatest on weekdays, and 
much lower on weekends. This pattern is somewhat different than that reported by previous research. In the study of complaints made to the OPP, Wickens et al. (2005) found that the number of reports tended to increase over the course of the week, peaking on Friday and Saturday. Report frequency was at its lowest point from Sunday to Tuesday. In their survey of organizations fighting aggressive-driving, Rathbone and Huckabee (1999) also found an increasing number of reports over the course of the week that was greatest on Fridays and was lowest on the weekends. In a content analysis of complaints made to the California Highway Patrol in San Diego, Sarkar et al. (2000) reported that the number of calls was greater than expected on Fridays and Wednesdays, but lower than expected on Sundays, Mondays, and Saturdays. In an analysis of charges laid by the Western Australian Police Service involving impulsive driving-related violence between strangers, Harding et al. (1998) found minimal differences over the course of the week; however, the greatest number of offences occurred on Fridays and the fewest number of offences occurred on Mondays.

Therefore, the RoadRagers.com data seems to have replicated the general finding of a decline in reports on weekends, but failed to replicate the finding of an increased number of reports on Fridays. This difference in results from that found in other archival studies may indicate a difference in the sample. It is possible that visitors to the RoadRagers.com website are primarily daytime workers, particularly workers with computer access. These individuals are most likely to be out on the roads during the weekdays, and can easily report offending drivers they have encountered during their morning commute or their afternoon commute the previous day when they arrive at work. If they encounter offensive drivers on the weekend, these workers may not have easy access to a computer or may not be willing to take the extra time to report these offenders.

It should also be noted that studies using other methodologies have identified alternative 
weekly patterns. For instance, in two driving diary studies examining levels of driver stress, a weekend effect was found whereby stress accumulated over the course of the week, peaked on Thursday evenings, and declined over the weekend (Gulian et al., 1990; Langford and Glendon, 2002).

\subsection{Hourly reporting trends}

The greatest number of RoadRagers.com complaints originated between 0600 and $0859 \mathrm{~h}$ and between 1500 and $1759 \mathrm{~h}$. These timeslots coincide with the standard morning and afternoon rush hours, adding further support to the contention that the sample included in this dataset consists primarily of daytime workers with computer access. This hypothesized difference in sample also helps to explain why the time of day results for the RoadRagers.com data is different than that of the OPP data (Wickens et al., 2005), which found that the number of reports tended to increase over the course of the day, peaking in the $1500-1800 \mathrm{~h}$ timeslot. It is possible that the daytime workers hypothesized to constitute the primary contributors to the RoadRagers.com website are willing to report morning commute altercations because these events are fresh in their memory when they arrive at work and power on their computers. Drivers may be less willing to telephone the OPP in the morning than they are to telephone them in the afternoon. Perhaps because drivers in the morning are more likely to have a specific time by which they must arrive at their destination and thus are experiencing more time urgency than they would in the afternoon, they may be less likely to telephone the police to report an offending driver.

Previous research has not been entirely consistent in terms of time of day results. The analysis of offences in Western Australia also reported an increasing number of reports over the course of the day, peaking in the 1500 - $1800 \mathrm{~h}$ timeslot (Harding et al., 1998), and the analysis of reports made to the California Highway Patrol concluded that the number of reports were greatest in the 1500 - 1800 h timeslot (Sarkar et al., 2000). However, Rathbone and Huckabee's 
(1999) survey of organizations combating aggressive driving found that the greatest number of incidents occurred during the afternoon rush hour, but there was also a less pronounced peak during the morning rush hour. It is possible that data from websites like RoadRagers.com may be providing additional information about morning commutes that is not available from police sources. This possibility is supported by the results of driver diary studies which have found that reported driver stress is only slightly greater during the evening than the morning commute, if at all (Gulian et al., 1990; Langford and Glendon, 2002).

\subsection{Implications for driver safety initiatives}

Individualized hierarchies of imagined roadway situations that provoke increasing degrees of anger have been used previously in desensitization exercises to assist with anger management training for aggressive drivers (Galovski et al., 2006). Results of the current study can be used in a similar fashion, educating drivers concerning how their own behaviours on the roadway can contribute to other drivers' anger and aggression. Knowledge of a hierarchy of increasingly offensive roadway behaviours may assist drivers to avoid consciously committing the most upsetting of these behaviours. Moreover, when applied early in the drivers' training, these lessons can go a long way in assisting motorists to avoid developing anger-provoking involuntary roadway habits that may be more difficult to expunge later (e.g., changing lanes without signaling).

A wide scale adoption of the coding system could improve the breadth and precision of data collection by policing agencies. For example, as noted previously, it is likely that personnel at the OPP Call Centre did not record incidents of driver hostility because obscene gestures and unkind language are not illegal and are irrelevant for officers in the field. The use of a definitive, reliable, and easily mastered coding scheme at the time a complaint is submitted to the OPP Call Centre would allow for more accurate recording of data and would provide an easily analyzed set 
of data to examine trends across jurisdictions and differing time frames. This data would provide information to supplement that provided by tickets for moving violations issued by police.

Wide-scale adoption of the coding system by policing agencies could also provide the basis for selecting improper driving behaviours to be targeted by news features, public service television commercials, discussions on talk radio by highway safety officials, etc. Once educational and public relations efforts are undertaken, the coding system could be applied to subsequent program evaluation data to gauge the effectiveness of the interventions by comparing pre-and post-intervention complaints.

\subsection{Future research directions}

Further improvements to driver safety could be made by understanding the motivations behind offensive driver behaviours. Although datasets like complaints to police departments or online websites do not provide insight into the offensive driver's motivation, online websites may provide insight into the victim driver's perceptions of the perpetrator's motivations. The victim driver's perceptions may be just as valuable in understanding the development of driver anger and subsequent retaliatory regression. Attributional theory, which focuses on how cognitive explanations for events and actions impact subsequent emotions and behavioural responses, has recently been demonstrated to be highly relevant to episodes of driver anger and retaliatiory aggression (Roseborough et al., 2011; Wickens et al., 2011). A limited content coding scheme focused on victim drivers' perceptions of the cause of offensive roadway behaviours was recently piloted on driving diaries, and identified perceptions of negligence as being the most frequently reported perceived motivation for negative driving events (Wickens et al., 2012). Lennon and Watson (2011) conducted interviews with drivers to better understand motivations for perpetrated retaliatory aggression, and identified "teaching other drivers a lesson" and "justified retaliation" as primary motivations for roadway retribution. Attributional training that teaches drivers to 
recognize cognitive biases in their own interpretations of others' roadway behaviour, as well as cognitions associated with their own roadway vengeance, may help to avoid or minimize driver anger and subsequent retaliatory aggression.

\subsection{Strengths and limitations of website data}

Results of the current study suggest that data from websites like RoadRagers.com have both their strengths and their weaknesses. The accuracy of the data itself is not as reliable as that of other data sources such as complaints submitted to and recorded by police departments. The RoadRagers.com website asks drivers to specify the time, day, month, and even year of the reported incident, but some drivers may not take the time and care needed to accurately report this information. We also know from drivers who did take extra effort to provide detailed information that the website experienced technical difficulties that may have affected the accuracy of the information provided by users. The complainants are also not representative of the general driving population, being restricted to drivers who are computer literate and have access to the Internet. This constitutes a selection bias, whereby the views of groups such as the poor, elderly, or uneducated are less represented in the sample of complaints. Moreover, we have no direct knowledge of the demographic characteristics of the sample beyond the reported state or province in which the driving event occurred. This greatly limits our ability to interpret the results of the analysis. Still, the complaints submitted to the website by drivers are not filtered by police personnel; thus these complaints may include detailed descriptions of driver behaviour (e.g., blocking and erratic braking) that might not be retained in police reports because they are not specified in highway traffic legislation.

Therefore, websites that solicit driver complaints of others' improper driver behaviour must be treated carefully as a data source. However, these websites represent a useful tool to confirm the findings from other data sources and to supplement data that may be missing from 
those other sources. The current study confirmed previous findings that cutting and weaving is the most frequently reported driver complaint (Sarkar et al., 2000; Wickens et al., 2005; 2012), followed by speeding. It also confirmed that these events are most frequently reported during high-traffic periods (Gulian et al., 1990; Harding et al. 1998; Langford and Glendon, 2002; Rathbone and Huckabee, 1999; Sarkar et al., 2000; Wickens et al., 2005). As with the content analysis of complaints to the OPP, the current content analysis of website complaints cannot determine whether cutting and weaving is the most frequently occurring offensive driver behaviour, or if it is perceived as the most offensive and thus more likely to be reported to the website.

\section{Conclusion}

To the best of our knowledge, this study conducted the first content analysis of an online complaint website, and identified both strengths and weaknesses of this new data source. Moreover, the study demonstrated the value of the content analysis methodology to driver research. Although content analysis has not been widely used by transportation researchers, it is clearly an important research tool. The current study also provided further validation of the driver behaviour coding scheme developed by Wickens et al. (2005). The reliability of the coding scheme as applied to the current dataset was very good, and the behavioural classification of driving events across studies was generally consistent. The temporal distribution of these events appeared to differ somewhat from previous studies; however, these differences are likely explained by the types of complainants accessing the RoadRagers.com website (i.e., likely daytime workers with computer access). Overall, this study successfully achieved each of its primary goals, and has important implications for both driver safety initiatives and future research directions. 


\section{Acknowledgements}

Dr. Wickens was supported by postdoctoral fellowships from the Social Sciences and Humanities Research Council and the Centre for Addiction and Mental Health. The authors would like to thank various anonymous reviewers for valuable recommendations that greatly improved the manuscript, as well as several undergraduate students who assisted with the significant amount of data entry necessary for this analysis: Vadim Paskarou, Arathi Packiyanathan, Meenu Minhas, Karmpaul Singh, Ramy Kirollos, and Eva Bernacka-Shiner. 


\section{References}

Deffenbacher, J.L., Deffenbacher, D.M., Lynch, R.S., Richards, T.L., 2003. Anger, aggression, and risky behavior: a comparison of high and low anger drivers. Behavior, Research and Therapy, 41, 701-718.

Deffenbacher, J.L., Oetting, E.R., Lynch, R.S., 1994. Development of a driving anger scale. Psychological Reports, 74, 83-91.

Galovski, T. E., Malta, L. S., Blanchard, E. B., 2006. Road rage: Assessment and treatment of the angry, aggressive driver, Washington, DC: American Psychological Association.

Groeger, J.A., 1997. Mood and driving: Is there an effect of affect?. In: T. Rothengatter \& E.C. Vaya (Eds.), Traffic and transport psychology: Theory and application, New York: Pergamon, pp. 335-342.

Gulian, E., Debney, L.M., Glendon, A.I., Davies, D.R., Matthews, G., 1989. Coping with driver stress. In: F.J. McGuigan, W.E. Sime, \& J.M. Wallace (Eds.), Stress and tension control 3: Stress management, New York: Plenum Press, pp. 173-186.

Gulian, E., Glendon, A.I., Matthews, G., Davies, D.R., Debney, L.M., 1990. The stress of driving: A diary study. Work \& Stress, 4, 7-16.

Harding, R.W., Morgan, F.H., Indermaur, D., Ferrante, A.M., Blagg, H., 1998. Road rage and the epidemiology of violence: Something old, something new. Studies on Crime and Crime Prevention, 7, 221-238.

Hennessy, D.A., Wiesenthal, D.L., 1999. Traffic congestion, driver stress, and driver aggression. Aggressive Behavior, 25, 409-423.

Hennessy, D.A., Wiesenthal, D.L., 2001a. Gender, driver aggression, and driver violence: An applied evaluation. Sex Roles, 44, 661-676. 
Hennessy, D.A., Wiesenthal, D.L., 2001b. Further validation of the Driving Vengeance Questionnaire. Violence and Victims, 16, 565-573.

Lajunen, T., Parker, D., 2001. Are aggressive people aggressive drivers? A study of the relationship between self-reported general aggressiveness, driver anger and aggressive driving. Accident Analysis and Prevention, 33, 243-255.

Langford, C., Glendon, A. I., 2002. Effects of neuroticism, extraversion, circadian type and age on reported driver stress. Work \& Stress, 16, 316-334.

Lennon, A., Watson, B., 2011. "Teaching them a lesson?” A quantitative exploration of underlying motivations for driver aggression. Accident Analysis and Prevention, 43, 2200-2208.

Matthews, G., Dorn, L., Glendon, A. I., 1991. Personality correlates of driver stress. Personality and Individual Differences, 12, 535-549.

Mesken, J., Hagenzieker, M.P., Rothengatter, T., De Waard, D., 2007. Frequency, determinants, and consequences of different drivers' emotions: An on-the-road study using self-reports, (observed) behaviour, and physiology. Transportation Research Part F, 10, 458-475.

National Highway Traffic Safety Administration, 1998, April. Final report - Capital Beltway update: Beltway user focus groups, downloaded July 24, 2001 from http://www.nhtsa.dot.gov/people/injury/research/aggressive/final.rpt.html.

Neighbors, C., Vietor, N.A., Knee, C.R., 2002. A motivational model of driving anger and aggression. Personality and Social Psychology Bulletin, 28, 324-335.

Nesbit, S.M., Conger, J.C., Conger, A.J., 2007. A quantitative review of the relationship between anger and aggressive driving. Aggression and Violent Behavior, 12, 156-176.

Novaco, R.W., 1991. Aggression on roadways. In: R. Baenninger (Ed.), Targets of violence and aggression, New York: Elsevier Science Publishers, pp. 253-326. 
Parker, D., Lajunen, T., Summala, H., 2002. Anger and aggression among drivers in three European countries. Accident Analysis and Prevention, 34, 229-235.

Parkinson, B., 2001. Anger on and off the road. British Journal of Psychology, 92, 507-526.

Rathbone, D.B., Huckabee, J.C., 1999, June 19. Controlling road rage: A literature review and pilot study, downloaded October 15, 2000 from http://www.aaafoundation.org/resources/index.cfm?button=roadrage.

Roseborough, J., Wiesenthal, D. L., Flett, G. L., Cribbie, R. A., 2011. The belief in an unjust world and narcissism - The influence of personality on perceptions of injustice, driving anger, and aggressive driving. Proceedings of the $21^{\text {st }}$ Canadian Multidisciplinary Road Safety Conference, Halifax, Nova Scotia, Canada.

Sarkar, S., Martineau, A., Emami, M., Khatib, M., Wallace, K., 2000. Aggressive driving and road rage behaviors on freeways in San Diego, California. Transportation Research Record, 1724, 7-13.

Shin, P.C., Hallett, D., Chipman, M.L., Tator, C., Granton, J.T., 2005. Unsafe driving in North American automobile commercials. Journal of Public Health, 27, 318-325.

Snyder, D.F., 1997, July 17. Statement of David F. Snyder, Assistant General Counsel, American Insurance Association, representing advocates for highway and auto safety before the Subcommittee on Surface Transportation, Committee on Transportation and Infrastructure, U.S. House of Representatives.

Tasca, L., 2000. A review of the literature on aggressive driving research. Paper presented at the Online Aggressive Driving Issues Conference, downloaded June 26, 2001 from http://www.aggressive.drivers.com/board/messages/25/49.html.

Underwood, G., Chapman, P., Wright, S., Crundall, D., 1999. Anger while driving. Transportation Research Part F, 2, 55-68. 
Vanlaar, W., Simpson, H., Mayhew, D., Robertson, R., 2008. Aggressive driving: A survey of attitudes, opinions and behaviors. Journal of Safety Research, 39, 375-381.

Whitley, B.E., Jr, 1996. Principles of research in behavioral science. Toronto: Mayfield Publishing Company.

Wickens, C. M., Roseborough, J. E. W., Hall, A., Wiesenthal, D. L., 2012. Dear diary... A content analysis of anger-provoking events reported in driving diaries. Proceedings of the $22^{\text {nd }}$ Canadian Multidisciplinary Road Safety Conference, Banff, Alberta, Canada.

Wickens, C.M., Wiesenthal, D.L., 2005. State driver stress as a function of occupational stress, traffic congestion, and trait stress susceptibility. Journal of Applied Biobehavioral Research, 10, 83-97.

Wickens, C.M., Wiesenthal, D.L., Flora, D.B., Flett, G.L., 2011. Understanding driver anger and aggression: Attributional theory in the driving environment. Journal of Experimental Psychology: Applied, 17, 354-370.

Wickens, C.M., Wiesenthal, D.L., Rippey, K., 2005. Motorists' perception of aggressive driving: A comparative analysis of Ontario and California drivers. In: D.A. Hennessy \& D.L. Wiesenthal (Eds.), Contemporary Issues in Road User Behavior and Traffic Safety, Hauppauge, NY: Nova Science Publishers, pp. 23-36.

Wiesenthal, D.L., Hennessy, D.A., Gibson, P.M., 2000. The Driving Vengeance Questionnaire (DVQ): The development of a scale to measure deviant drivers' attitudes. Violence and Victims, 15, 115-136. 


\section{Table 1}

Coding scheme (abbreviated)

(A) Improper speed

(i) Speeding/racing

(ii) Unnecessary slow driving

(iii) Sporadic speeds

(B) Tailgating

(C) Lane usage

(i) Improper lane usage

(ii) Weaving through traffic/cutting off/sideswiping

(D) Improperly equipped/unsafe vehicle

(i) No turn signal

(ii) All other forms of improperly equipped/unsafe vehicle

(E) Disobedience of traffic signs/signals

(F) Erratic/improper braking

(G) Blocking

(H) Perceived driver displays of hostility or violence

(i) Perceived hostile driver displays

(ii) Perceived violent driver displays
Speed greater than the posted limit or too high for the current road conditions

Such a slow rate of speed as to impede the normal movement of traffic

Continuously changing or unpredictable speeds

Following a vehicle more closely than is reasonable

Has not driven within a single lane, has passed another vehicle by driving outside a single lane and onto the shoulder of the roadway, or has violated the legally prescribed use of a lane Has moved from one lane to another or has entered a lane from a side road without due regard for whether that movement can be made safely

Failed to use a flashing light signal for turn or lane change Has operated a vehicle that is improperly equipped (e.g., improper display of license plate and lack of headlight/tail light) or is in such an unsafe condition as to potentially endanger others (e.g, mechanical error and overloading) Failed to obey a traffic control sign (e.g., red light, yield sign, pedestrian crossing)

Sudden and unnecessary braking without due regard for other vehicles

Preventing other vehicles from merging or passing

E.g., yelling, gesturing, horn honking, and flashing high beams

Behavioural displays of discontent that are more intense than verbal commentary or gestures that are not included elsewhere in the coding scheme (e.g., chasing/following, getting out of the vehicle to verbally or physically argue, waving a firearm/blunt instrument/weapon, and throwing threatening objects) 

(i) Cell phone use
Inattention associated with cell phone use
(ii) Other or unspecified distraction
Source of inattention not specified or associated with anything but a cell phone
(J) Hazardous road conditions not
E.g., people/hitchhikers on the road, attributable to driver behaviour disabled/abandoned vehicle, and debris on the road
(K) Cannot be classified
Cannot be placed in any of the above categories 
Fig. 1. Frequency of RoadRagers.com complaints by category.

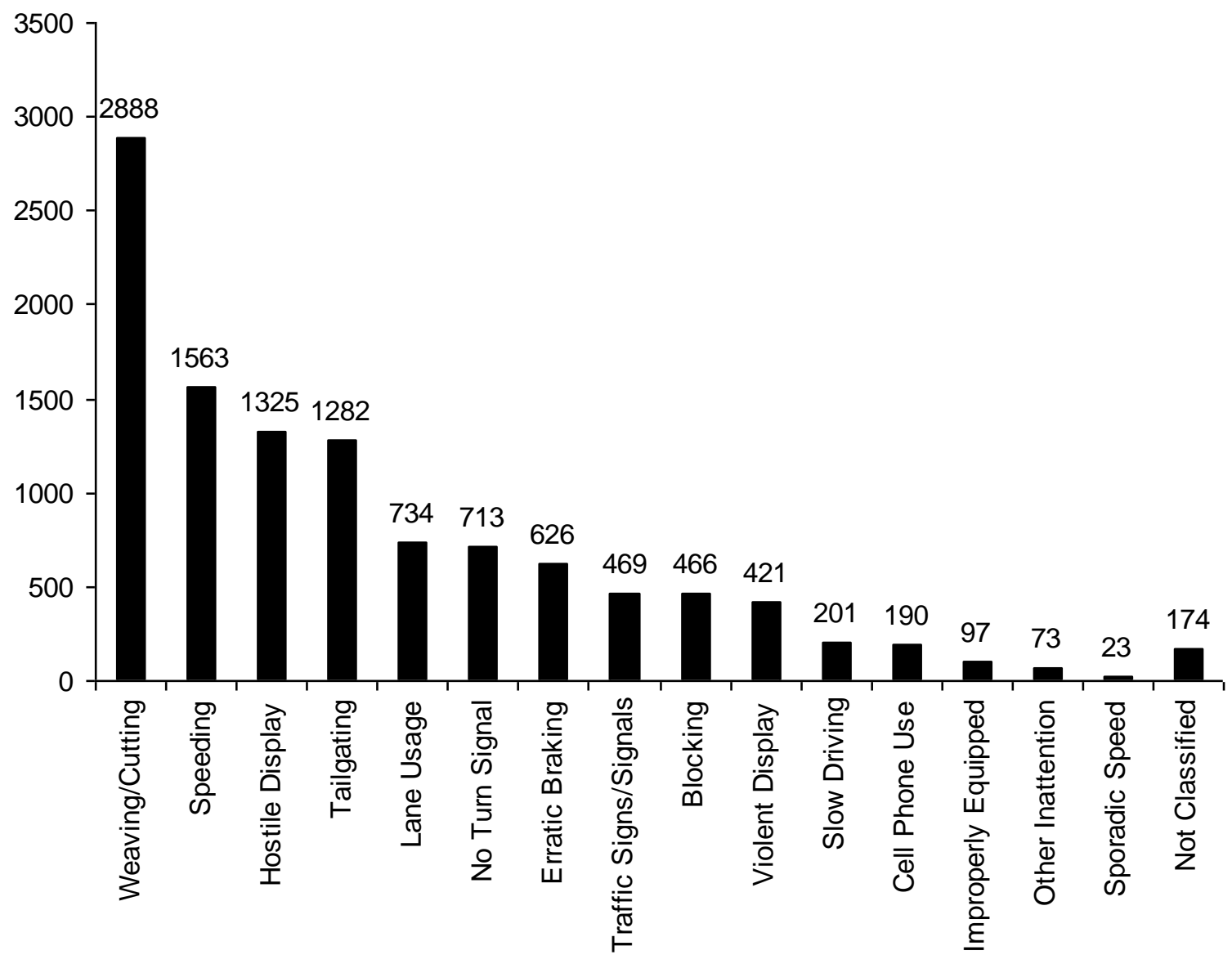

Categories 
Fig. 2. Frequency of RoadRagers.com complaints by month of year.

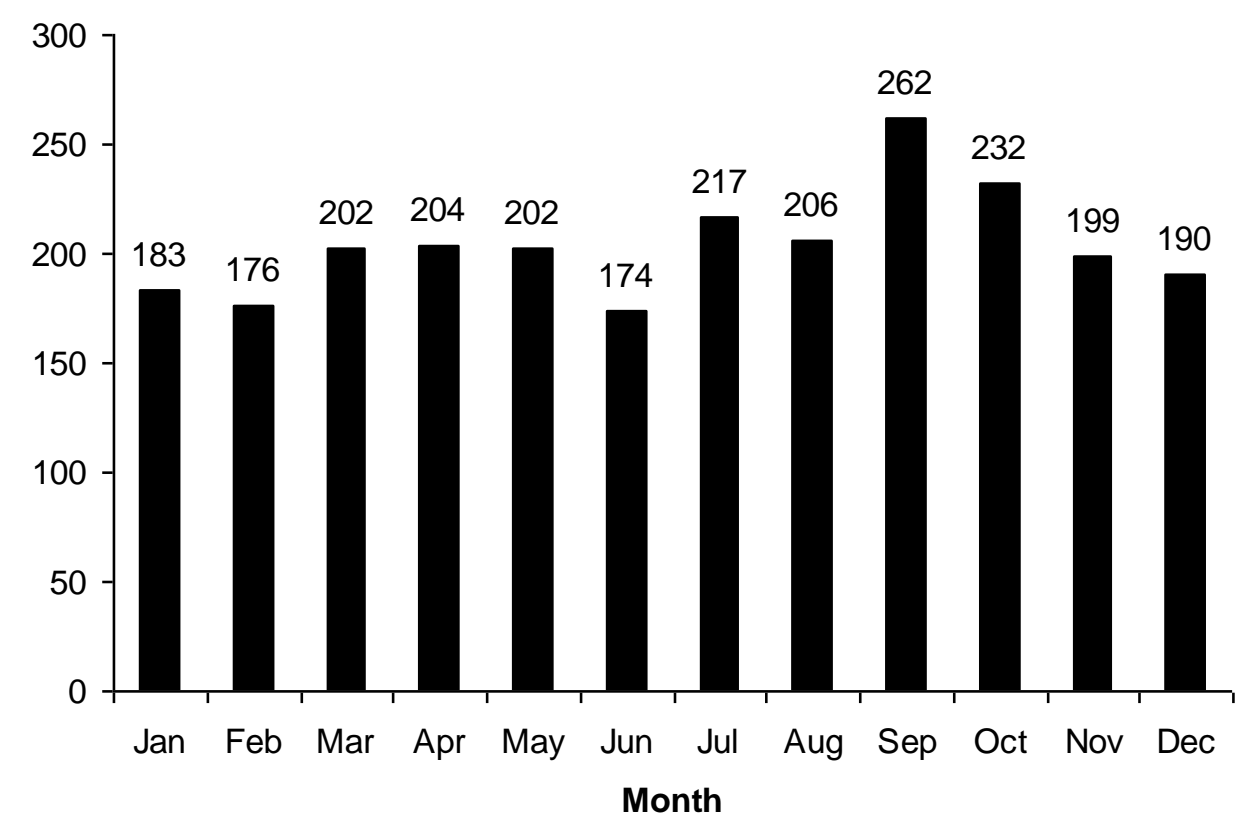


Fig. 3. Frequency of RoadRagers.com complaints by day of the week.

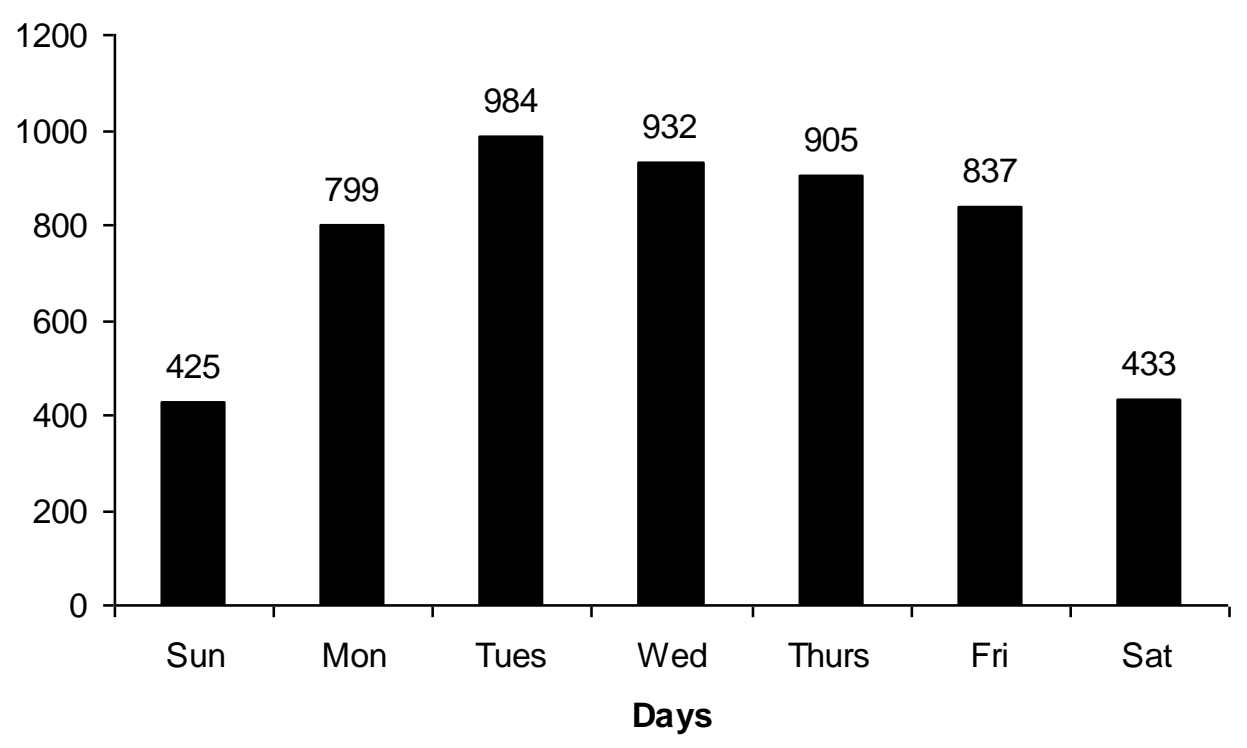


Fig. 4. Frequency of RoadRagers.com complaints by time of the day.

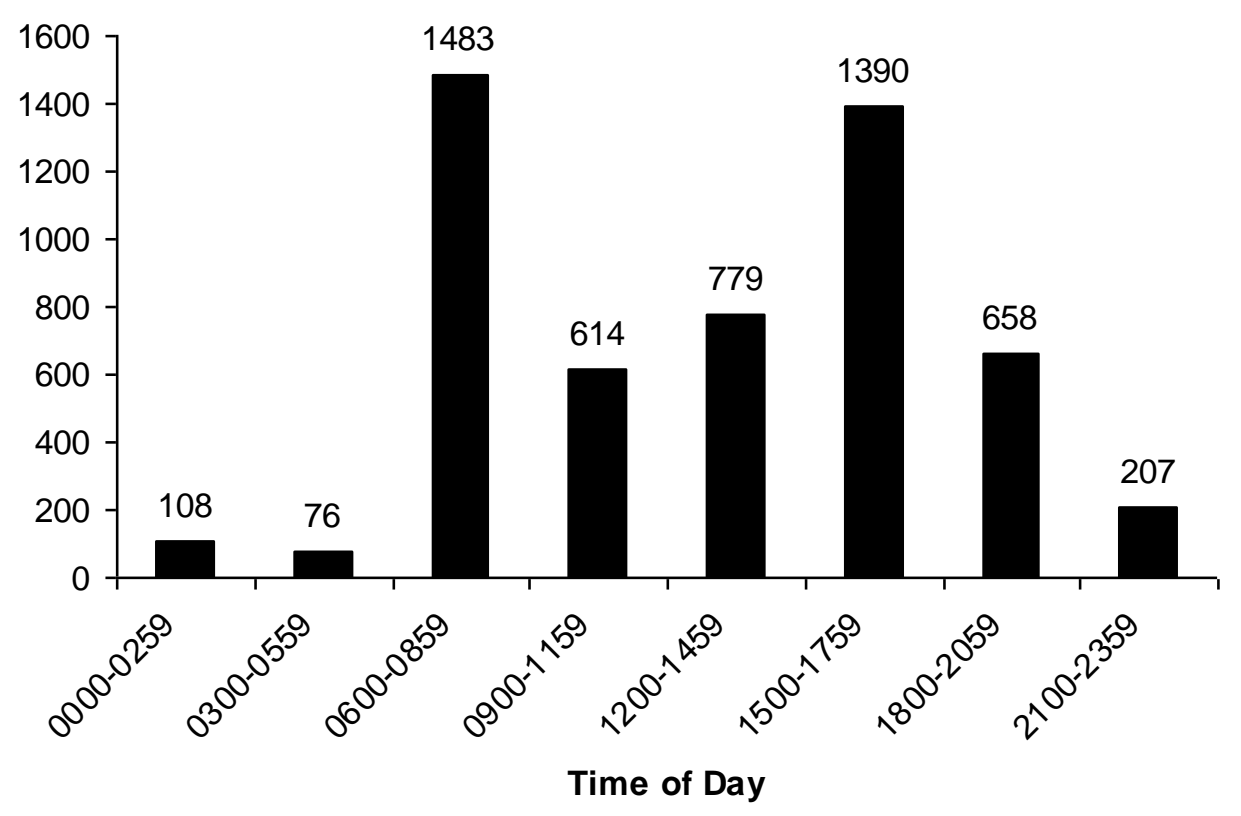

